\title{
Identification of ELAVL4 as a modulator of radiation sensitivity in A549 non-small cell lung cancer cells
}

\author{
KYU JIN CHOI ${ }^{1 *}$, JI-HYUN LEE $^{1,2 *}$, KWANG SEOK KIM $^{1}$, \\ SEONGMAN KANG ${ }^{2}$, YUN-SIL LEE ${ }^{3}$ and SANGWOO BAE ${ }^{1}$ \\ ${ }^{1}$ Laboratory of Modulation of Radiobiological Responses, Korea Institute of Radiological and Medical Sciences (KIRAMS), \\ Seoul 139-706; ${ }^{2}$ School of Life Sciences and Biotechnology, Korea University, Seoul 136-701; \\ ${ }^{3}$ Laboratory of radiation damage control, KIRAMS, Seoul 139-706, Republic of Korea
}

Received November 19, 2010; Accepted January 10, 2011

DOI: $10.3892 /$ or.2011.1260

\begin{abstract}
Identification of genes that modulate radiation sensitivity provides important tools to study cellular responses to ionizing radiation. We combined DNA microarrays and viability assays to identify modulators of radiation sensitivity in A549 lung cancer cells. Up-regulated genes were selected from microarray experiments and RNA expression levels were confirmed by real-time RT-PCR analysis. Cell viability assays such as clonogenic assay, MTT and FACS analysis of cell death, identified the ELAVL4 gene as a novel modulator of radiation sensitivity. ELAVL4 expression was induced following ionizing irradiation. Depletion of the ELAVL4 gene increased radiation sensitivity of A549 cells as shown by decreased surviving cell fraction following irradiation in clonogenic assay. Enhanced radiation sensitivity of ELAVL4-depleted cells was attributable to decreased cell proliferation as well as increased apoptotic cell death following irradiation. Thus the endogenous function of ELAVL4 in relation to radiation sensitivity might be the regulation of cell proliferation and death. This approach to identification of modulators for radiation sensitivity has several advantages in terms of functional selectivity, stringency and time. Further analysis of the modulators should find potential use in the application of radiation biomarkers as well as modulators of cellular radiation responses.
\end{abstract}

\section{Introduction}

Ionizing radiation induces diverse cellular responses such as DNA damage repair, gene expression, cell cycle arrest

Correspondence to: Dr Sangwoo Bae, Laboratory of modulation of radiobiological responses, Korea Institute of Radiological and Medical Sciences (KIRAMS), 75 Nowon-gil, Nowon-gu, Seoul 139-706, Republic of Korea

E-mail: swbae@kcch.re.kr

* Contributed equally

Key words: radiation sensitivity, ELAVL4, microarray, non-small cell lung cancer and death (1). DNA damage caused by ionizing radiation is severe initiating cellular responses by triggering DNA damage signaling. Once early DNA damage response factors (DDRF) such as MDC1, 53BP1 and MRN complex recognize the damage, the factors facilitate activation of ATM kinase (2). ATM affects the cellular responses to ionizing radiation by transmitting DNA damage signaling to various downstream protein targets. For example, ionizing radiation induces apoptotic cell death via a pathway that involves activation of ATM, Chk2, p53 and expression of PUMA (3). G2/M phase cell cycle checkpoint is induced upon ionizing irradiation via regulation of $\mathrm{CDC} 2$ by a signaling pathway mediated by ATM, Chk1/Chk2 and CDC25 (1). As these cellular processes determine cellular viability in response to DNA damage, regulators of the processes are potential modulators of radiation sensitivity. Moreover, as the modulators could be targeted for enhanced radiation sensitivity or for therapeutic intervention, identification of the modulators is of importance. Accordingly many different approaches were employed to screen genes whose alterations can change sensitivity to ionizing radiation or to DNA damaging agents (4-9). These approaches mainly use large scale screening systems in yeast or in human cells following gene knockout or depletion. As alternative methods, gene expression profiles have been examined in response to DNA damage to gain understanding of regulators of DNA damage responses $(10,11)$. For the purpose of gene expression profiling following DNA damage, microarray approach has been widely used $(12,13)$. The results often show varied gene expression depending on the dose, time after irradiation, genetic background of cells, or cell type. Thus identification of genetic determinants of radiation sensitivity by the microarray methods might require additional functional studies for the potential regulators of cellular radiation responses.

Non-small cell lung cancer (NSCLC) cell lines are generally less sensitive to ionizing radiation (14). The PI3K and Akt signaling pathway has been associated with the radiation resistance phenotype of NSCLC (15-17). In addition genes such as TSPYL5, livin and Mcl-1 were identified as regulators of NSCLC in response to DNA damaging agents (18-20). Since cellular radiation sensitivity is determined by combinatorial effect of diverse cellular responses, 
Table I. The genes in real-time RT-PCR analysis. ${ }^{a}$

\begin{tabular}{llll}
\hline & Gene symbol & \multicolumn{1}{c}{ Description } & GenBank accession ID \\
\hline 1 & FBXL6 & F-box and leucine-rich repeat protein 6 & NM_024555 \\
2 & PPP3CA & Protein phosphatase 3 (formerly 2B), catalytic subunit, $\alpha$ isoformcarcineurin & BC025714 \\
3 & MOCS1 & Molybdenum cofactor synthesis 1 & NM_005942 \\
4 & ELAVL4 & ELAV (embryonic lethal, abnormal vision, Drosophila) -like 4 & AY033995 \\
5 & TMPRSS7 & Transmembrane protease, serine 7 & AK131211 \\
6 & ID1 & Inhibitor of DNA binding 1 & NM_181353 \\
7 & ZNF239 & Zinc finger protein 239 & NM_005674 \\
8 & CDCA7 & Cell division cycle associated 7 & BC027966 \\
9 & DEAF1 & Deformed epidermal autoregulatory factor 1 & NM_021008 \\
\hline
\end{tabular}

${ }^{\mathrm{a} A b b r e v i a t e d ~ g e n e ~ s y m b o l s ~ a n d ~ t h e i r ~ f u l l ~ n a m e s ~ a r e ~ s h o w n ~ w i t h ~ G e n B a n k ~ a c c e s s i o n ~ I D . ~}$

the existence of hitherto unknown modulators for radiation responses are expected in NSCLC.

We used an NSCLC cell line, A549 to identify novel modulators of radiation sensitivity. We reasoned that certain differentially expressed genes following ionizing radiation might play roles in later radiation responses which might affect radiation sensitivity. If so, we might be able to select those genes from data of DNA microarray and test the genes in radiation sensitivity by using simple viability assays.

Through these processes we selected ELAVL4 as a novel modulator of radiation sensitivity in A549 cells. ELAVL4 depletion alone decreased A549 cell proliferation. The ELAVL4-depleted cells exhibited enhanced radiation sensitivity as shown by decreased clonogenic survival and increased apoptotic cell death. Thus endogenous function of ELAVL4 in radiation responses of A549 cells seems to be associated with regulation of cell proliferation and death.

\section{Materials and methods}

Cell culture, irradiation and viability assays. Human nonsmall cell lung cancer cell (NSCLC) line A549 cells were grown in RPMI-1640 (Lonza, Walkersville, MD, USA) supplemented with $10 \%$ fetal bovine serum (Lonza, Walkersville), $100 \mathrm{U} / \mathrm{ml}$ penicillin, and $100 \mu / \mathrm{ml}$ streptomycin at $37^{\circ} \mathrm{C}$ in a humidified atmosphere of $5 \% \mathrm{CO}_{2}$ incubator. Cells were exposed to $\gamma$-ray from ${ }^{137} \mathrm{Cs} \gamma$-ray source (Atomic Energy of Canada, Ltd., Canada) with dose rate of $3.81 \mathrm{~Gy} / \mathrm{min}$.

Cell viability was measured using the 3-(4,5-dimethylthiazol-2-yl)-2,5,-diphenyltetrazolium bromide (MTT) conversion assay in a 24-well plate. The culture medium was replaced with $1 \mathrm{ml}$ medium containing $0.5 \mathrm{mg} / \mathrm{ml}$ MTT (Sigma-Aldrich, St. Louis, MO, USA) and incubated for $60 \mathrm{~min}$ at $37^{\circ} \mathrm{C}$. Tetrazolium crystals were solubilized with $200 \mu 1$ DMSO. The absorbance was read at $595 \mathrm{~nm}$ in a micro-plate reader (Bio-Rad, Richmond, USA).

Clonogenic assay was performed by following standard protocols (21). To measure cell death, A549 cells were incubated for $72 \mathrm{~h}$ following ionizing irradiation. Cells were washed twice with cold PBS, trypsinized and stained with propidium iodide and Annexin V by using FITC Annexin V Apoptosis Detection Kit I (BD Biosciences, San Diego, CA, USA) for $15 \mathrm{~min}$ at room temperature in the dark. Fluorescence was measured with a flow cytometer (BD Biosciences, Franklin Lakes, NJ, USA) and the data obtained were analyzed with CellQuest software (BD Biosciences).

Transfection of cells with small interfering RNA. Pre-designed siRNA for ELAVL4 (cat. no. 1046881 Duplex), TMPRSS7 (cat. no. 1439392 Duplex) and negative control siRNA were purchased from Bioneer, Inc. (Daejeon, Korea). siRNA (100 nM) was transected into A549 cells using Lipofectamine ${ }^{\mathrm{TM}} 2000$ (Invitrogen, Carlsbad, USA) according to the manufacturer's instruction.

RNA extraction, microarray and data analysis. RNA was extracted from A549 cells by using TRI Reagent (Molecular Research Center Inc., OH, USA) according to the manufacturer's protocol. Microarray experiment was performed by Genocheck, Co., Ltd. (Gyeonggi-do, Korea). Briefly each total RNA sample $(10 \mu \mathrm{g})$ was labeled with cyanine (Cy5)conjugated dCTP (Amersharm, Piscataway, NJ, USA) by a reverse transcription reaction using reverse transcriptase, SuperScript II (Invitrogen). The Cy5-labeled cDNAs were resuspended in $10 \mu \mathrm{l}$ of hybridization solution (GenoCheck) and hybridized to Roche NimbleGen Human whole genome 12-plex array (Roche NimbleGen, Inc., WI, USA). Analysis of the arrays was done by using Axon GenePix 4000B scanner (Molecular Devices Corp., Sunnyvale, CA, USA) and software program of NimbeScan Version 2.4 (Roche NimbleGen).

Real-time quantitative RT-PCR analysis. To provide independent confirmation of microarray data, real-time quantitative reverse transcription-PCR was performed for the following genes, i.e., FBXL6, PPP3CA, MOCS1, ELAVL4, TMPRSS7, ID1, ZNF239, CDCA7 and DEAF1 (specification of these genes is listed in Table I). Used primers are listed in Table II. Real-time PCR analysis was performed using One Step SYBR PrimeScript $^{\mathrm{TM}}$ RT-PCR Kit II (Takara, Osaka, Japan) and DNA 
A

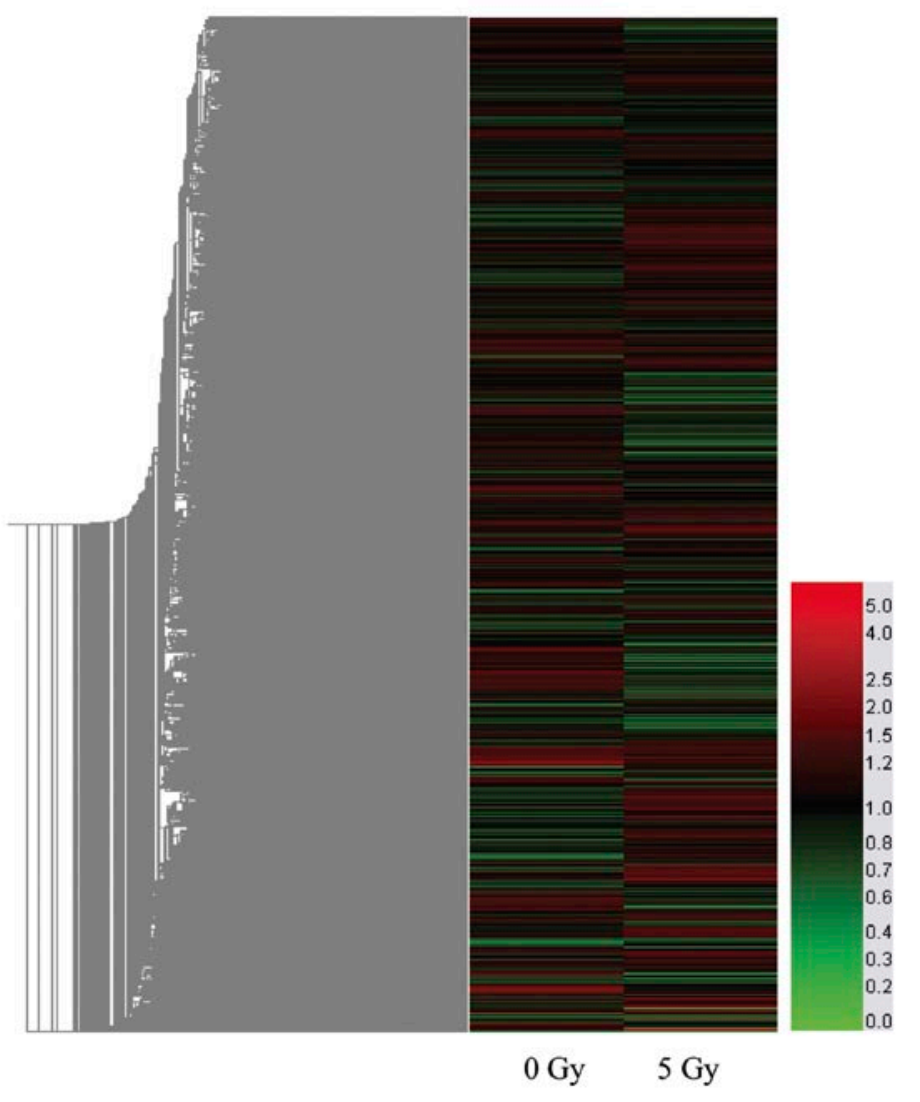

B

\begin{tabular}{|c|c|c|c|}
\hline & Significant over & Significant down & Total \\
\hline $\begin{array}{c}\text { A549 vs. } \\
\text { A549 5 Gy, 12 h }\end{array}$ & 1330 & 1307 & 2637 \\
\hline
\end{tabular}

Figure 1. DNA microarray was done with samples from A549 cells. Cells were non-irradiated or irradiated with 5 Gy of ionizing radiation (IR). After 12 h of incubation after IR, cells were harvested and RNA was isolated for DNA microarray. (A) Hierarchical clustering of total genes from non-irradiated and irradiated A549 cells. (B) Numbers of differentially expressed genes. Genes were sorted based on their expression levels either 2-fold up- or down-regulated.

Engine2.OPTICON (MJ Reserch, MA, USA). PCR reactions were done by following manufacturer's instruction (One Step SYBR PrimeScript RT-PCR Kit II). The relative expression of genes was normalized to reference gene, GAPDH.

\section{Results}

As changes in gene expression following ionizing radiation are responsible for the ensuing cellular responses, we carried out DNA microarray assay to identify genes that are responsible for modulation of radiation sensitivity. We examined gene expression profiles of A549 cell line, a non-small cell lung cancer cell line (NSCLC) after ionizing irradiation. After $12 \mathrm{~h}$ following $5 \mathrm{~Gy}$ of ionizing irradiation, the gene expression profiles were compared with non-irradiated control sample. Hierarchical clustering of total genes revealed that non-irradiated control and irradiated samples show significant differences
(Fig. 1A). When the genes were sorted based on 2-fold differences in expression levels either up- or down-regulated, $>1300$ up-regulated and equal number of down-regulated genes were identified from DNA microarray (Fig. 1B). Major functional categories of the differentially expressed genes include signal transduction, transcription, immune responses, cell cycle or differentiation (Fig. 2). In addition, apoptosis, cell proliferation, growth or other cellular processes are also observed.

As the purpose of the investigation was to identify genes that are responsible for radiation sensitivity, we selected several genes for further investigation (Table I). Especially we selected genes from up-regulated genes because their gene function in radiation sensitivity could be easily examined by gene depletion experiments. Expression levels of FBXL6, PPP3CA, MOCS1, ELAVL4, TMPRSS7, ID1, ZNF239, CDCA7 and DEAF1 were examined by real-time reverse transcription polymerase chain reaction (RT-PCR) with specific sets of 
Table II. Primer sets for quantitative reverse transcription polymerase chain reaction (RT-PCR) of the selected genes. ${ }^{\mathrm{a}}$

\begin{tabular}{|c|c|c|}
\hline & Gene symbol & Primer sequence \\
\hline 1 & FBXL6 & $\begin{array}{l}\text { Forward: CTCCGGGACGAGGGGTGGCT } \\
\text { Reverse: AGGGGCTGCCCTCCTTGGCT }\end{array}$ \\
\hline 2 & PPP3CA & $\begin{array}{l}\text { Forward: CTGCCTTCCCCTGGCTGCCC } \\
\text { Reverse: GGGCTTCGTGGGCTCGGAGT }\end{array}$ \\
\hline 3 & MOCS1 & $\begin{array}{l}\text { Forward: CTGGCCCGGCTACTGCCCCA } \\
\text { Reverse: GCGCACATCCAGGGGGAGGC }\end{array}$ \\
\hline 4 & ELAVL4 & $\begin{array}{l}\text { Forward: GGGCTGAATGGCCAGAAGCCCAG } \\
\text { Reverse: GGGGAGAACCTGGGGGAACAAGC }\end{array}$ \\
\hline 5 & TMPRSS7 & $\begin{array}{l}\text { Forward: TGGCTGGGGGCGAAGACACGA } \\
\text { Reverse: TGGTCGTCCACATCCATGTCCCCA }\end{array}$ \\
\hline 6 & ID1 & $\begin{array}{l}\text { Forward: CGCCTGCCTGCCCTGCTGGA } \\
\text { Reverse: CATGCCGCCTCGGCCGTCAG }\end{array}$ \\
\hline 7 & ZNF239 & $\begin{array}{l}\text { Forward: TGCACATCCACCAGCGAGTCCA } \\
\text { Reverse: TGCGAAGATCCGAGCTCTGGCTGA }\end{array}$ \\
\hline 8 & CDCA7 & $\begin{array}{l}\text { Forward: TGCCCAGAAGCCGTCGCTCCA } \\
\text { Reverse: AGGCGGGCAATGCCAGTTCGG }\end{array}$ \\
\hline 9 & DEAF1 & $\begin{array}{l}\text { Forward: CCCCTCTGGCTCCCGGCCAA } \\
\text { Reverse: CACTGCAAGGGTCGGCCCGC }\end{array}$ \\
\hline
\end{tabular}

${ }^{\mathrm{a}}$ Forward and reverse primers are indicated.

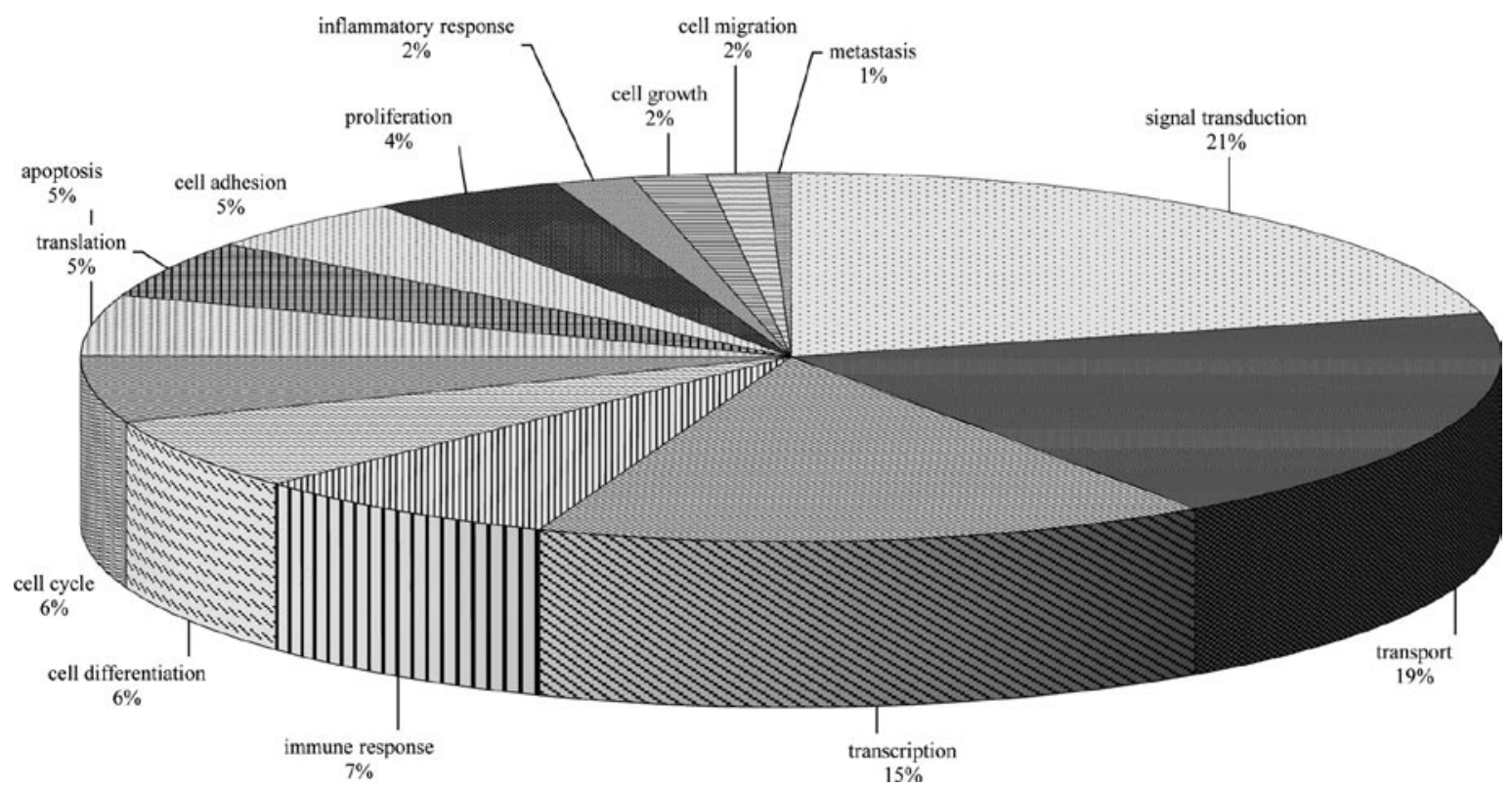

Figure 2. Functional categories of the differentially expressed genes after ionizing radiation. Categories were determined based on known cellular functions of the genes.

primers (Table II). Those genes are associated with cellular functions in metabolism, cell death, tumor suppression, or cell proliferation which might affect radiation sensitivity. Significant up-regulation of PPP3CA, ELAVL4, TMPRSS7 and ID1 was observed in irradiated A549 samples when compared to non-irradiated samples (Fig. 3).
In order to examine the genes as modulators of radiation sensitivity, we examined effect of specific gene depletion on cellular viability following ionizing radiation (Fig. 4). Depletion study of only ELAVL4 and TMPRSS7 are shown for comparison (other genes did not exhibit significant differences in radiation sensitivity, data not shown) (Fig. 4). When 


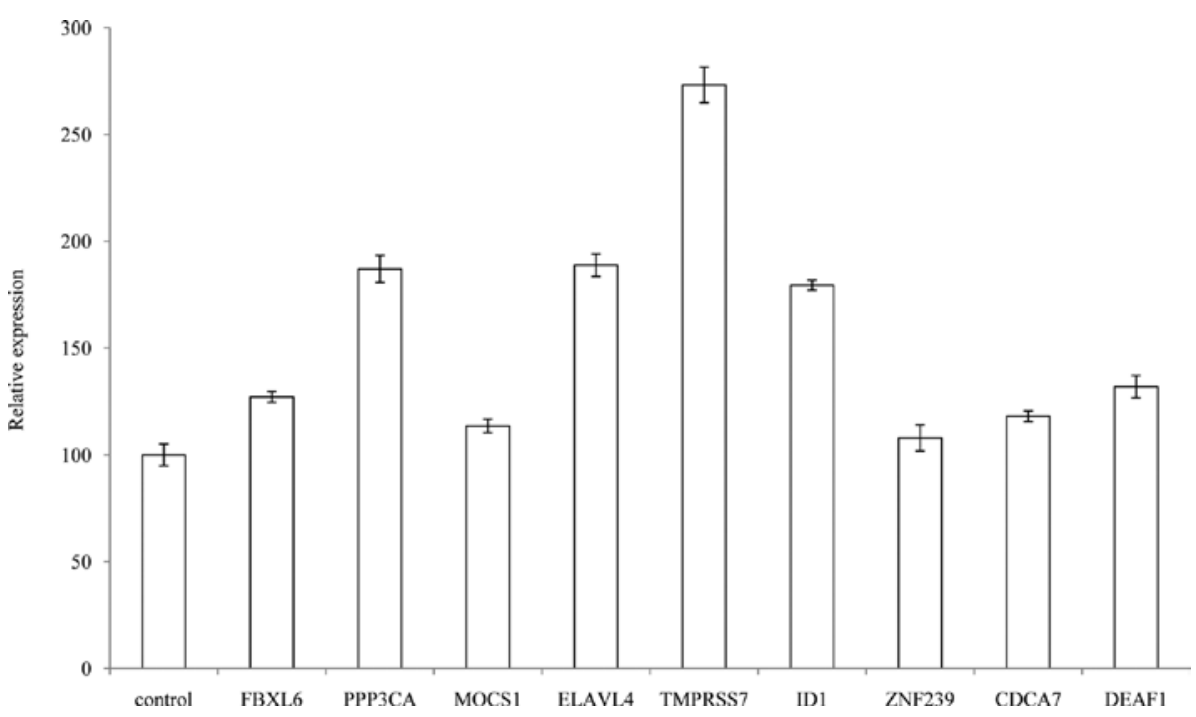

Figure 3. Analysis of the gene expression following ionizing radiation by real-time RT-PCR. A549 cells were either non-irradiated or irradiated with 5 Gy. Cells were harvested after $12 \mathrm{~h}$ and RNA was isolated for real-time RT-PCR. Real-time RT-PCR was done more than four times and standard deviation is indicated in the bar of the graph. Levels of gene expression in non-irradiated cells were set at $100 \%$ as a control. Gene expression of irradiated cells was compared to control.

A

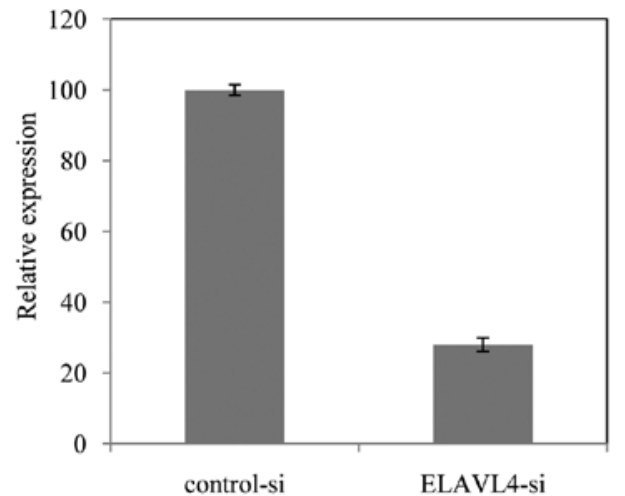

C

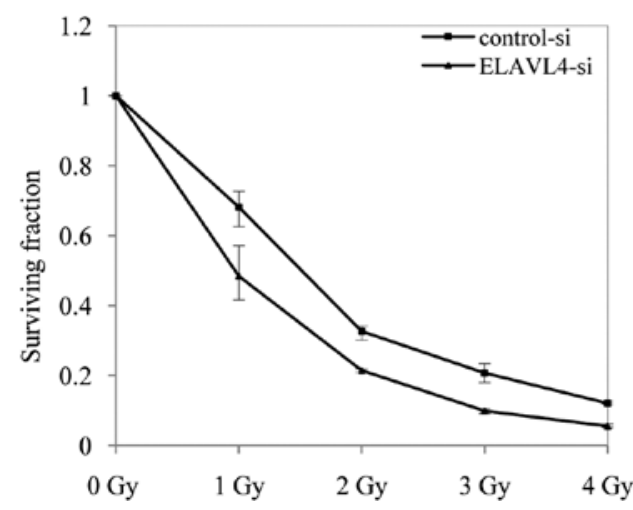

B

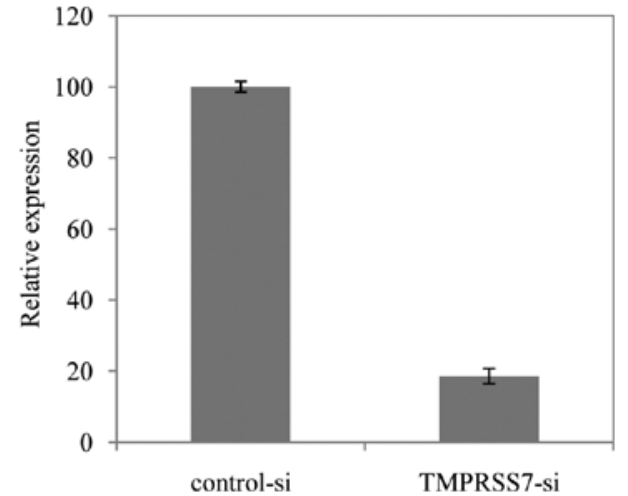

D

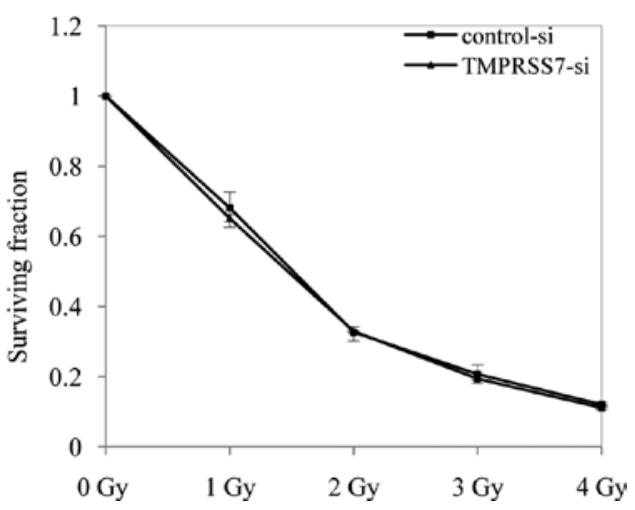

Figure 4. Effect of gene depletion on radiation sensitivity. Depletion of ELAVL4 (A) and TMPRSS7 (B) in A549 cells. Expression levels of the genes after depletion by siRNA were determined by real-time RT-PCR. For depletion of the gene expression, A549 cells were incubated with siRNA for $72 \mathrm{~h}$. Real-time RT-PCR was done more than four times. Standard deviation is indicated on each bar of the graph. (C) Clonogenic assay of A549 cells which were depleted of ELAVL4. A549 cells were treated with control siRNA or ELAVL4 siRNA and incubated for $24 \mathrm{~h}$. After splitting of the siRNA-treated cells and incubation for another $24 \mathrm{~h}$, cells were irradiated with $0,1,2,3$ and $4 \mathrm{~Gy}$ of IR. Number of colonies were counted and expressed in comparison to 0 Gy control sample which was set as 1 in y-axis. Four separate clonogenic assays were performed. Standard deviation is also indicated in the graph. (D) Clonogenic assay of A549 cells depleted of TMPRSS7. siRNA treatment and irradiation (C). Clonogenic assay shown as a graph (C).

A549 cells were depleted of $>70 \%$ of ELAVL4 or TMPRSS7, colony forming ability of the cells was examined following irradiation with various doses (Fig. 4). Decreased expression of ELAVL4 by siRNA depletion exhibited enhanced radiation 
A

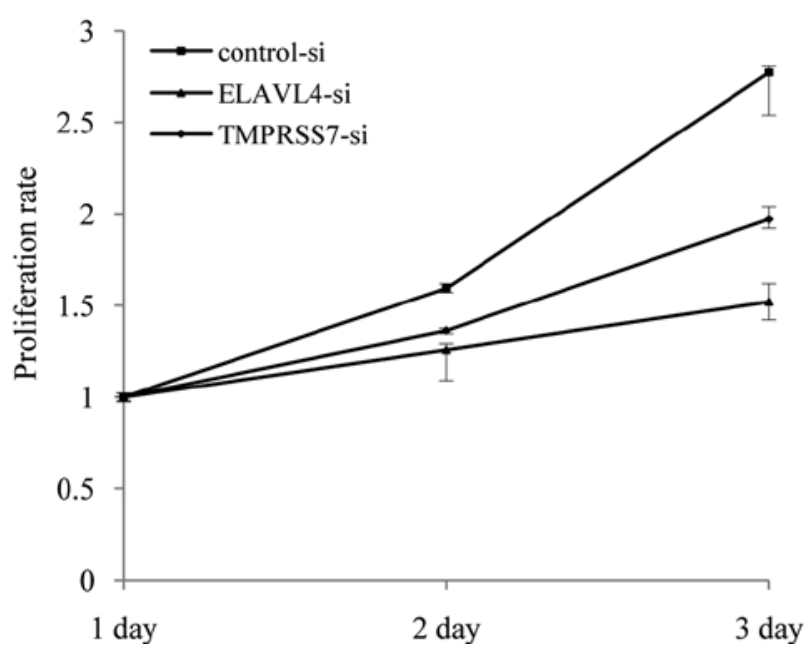

B

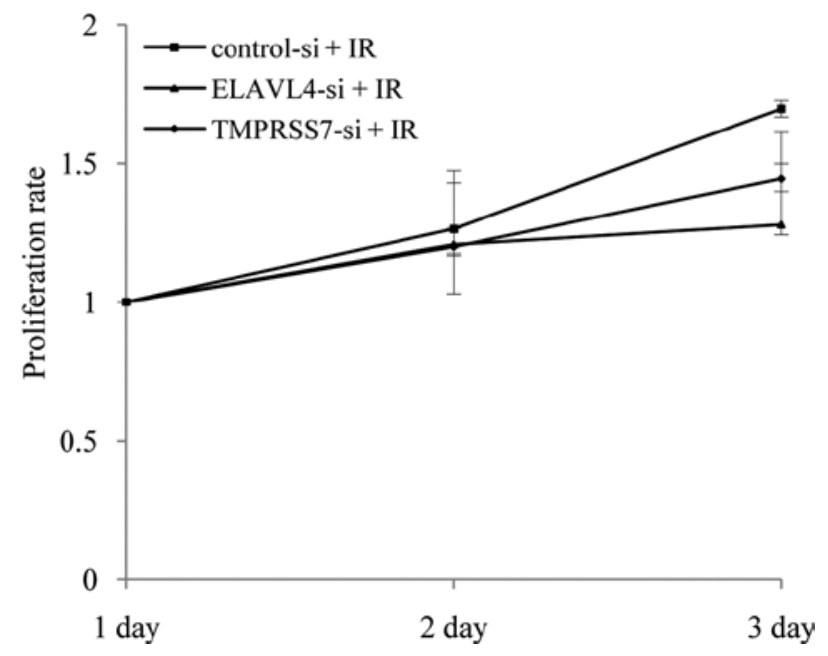

C

IR
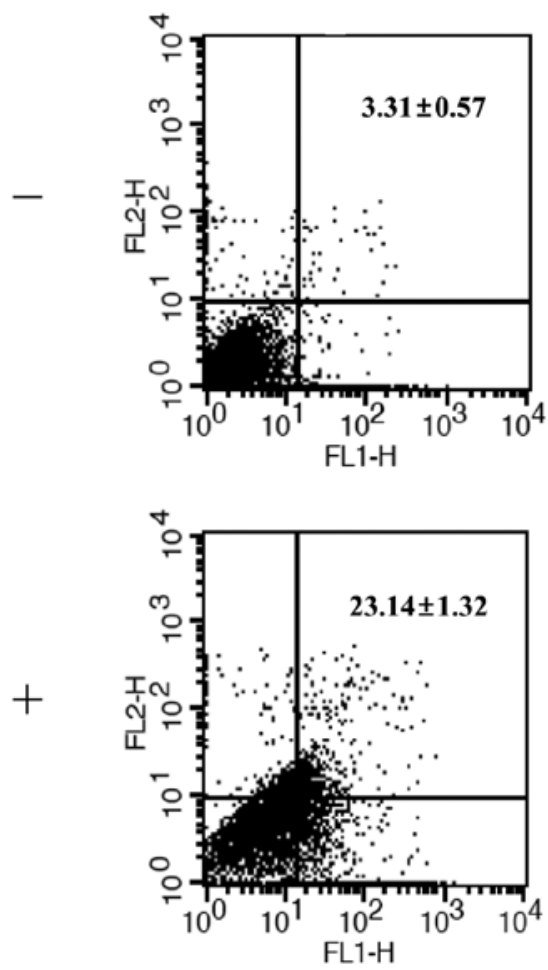

control-si
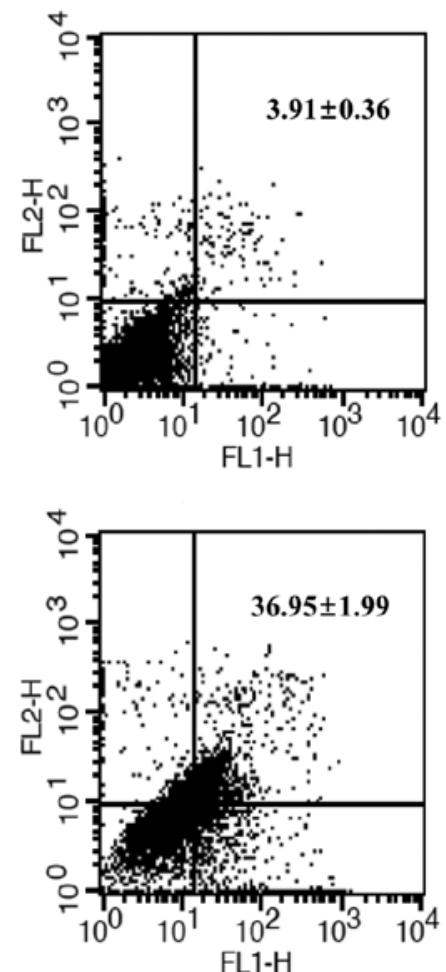

TMPRSS7-si
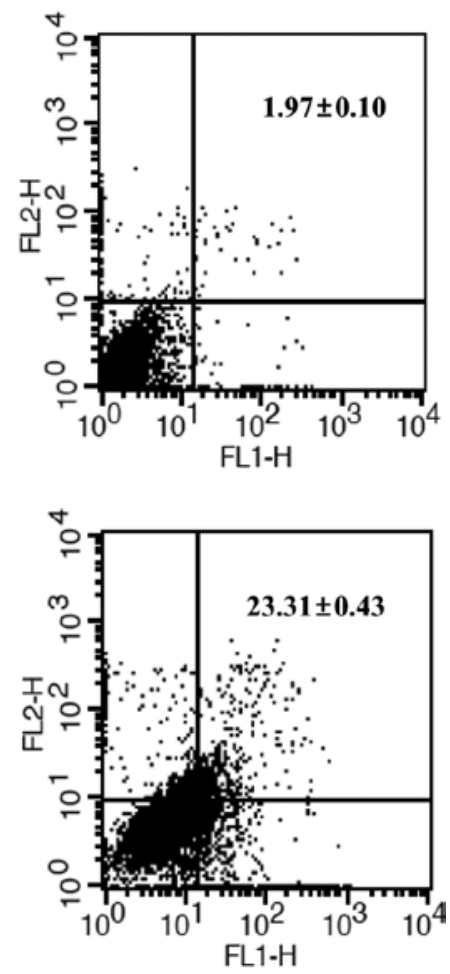

Figure 5. Effect of ELAVL4 or TMPRSS7 gene depletion on cell proliferation and apoptotic cell death. (A) MTT assay of control siRNA-treated, ELAVL4or TMPRSS7-depleted A549 cells in the absence of ionizing radiation. After the gene depletion, cell proliferation was measured by MTT assay after 1,2 and 3 days. Four separate MTT assays were performed. Standard deviation is indicated in the graph. Proliferation at day 1 is set as 1 and proliferation at days 2 and 3 were normalized to that of day 1. (B) Effect of IR on proliferation of control siRNA-treated, ELAVL4- or TMPRSS7-depleted A549 cells. After $24 \mathrm{~h}$ of the gene depletion, the cells were plated onto 24-well plates. After another $24 \mathrm{~h}$, cells were irradiated with $10 \mathrm{~Gy}$ of ionizing radiation. Cell proliferation was measured by MTT assay after 1,2 and 3 days. Four separate MTT assays were performed. Standard deviation is indicated in the graph. Proliferation at day 1 is set as 1 and proliferation at days 2 and 3 were normalized to that of day 1. (C) Apoptotic cell death measurement of control siRNA-treated, ELAVL4- or TMPRSS7-depleted A549 cells in the absence or presence of ionizing radiation. A549 cells were treated with the respective siRNA as in (A and B). The cells were irradiated with $10 \mathrm{~Gy}$ and incubated for $72 \mathrm{~h}$. Apoptotic cell death was measured by FACS after staining cells with propidium iodide and annexin V. Three separate measurements were done and the average and standard deviation for each condition is indicated.

sensitivity by showing less surviving cell fraction whereas TMPRSS7 depletion did not affect radiation sensitivity. These results indicate that ELAVL4 modulates radiation sensitivity in A549 lung cancer cells and that decreased ELAVL4 expression render the lung cancer cells sensitive to ionizing radiation. Because clonogenic assay tests cellular ability to undergo division, the results following ionizing radiation could result from either cell proliferation or death. We examined the 
cause of the enhanced radiosensitivity by ELAVL4 depletion. Effect on cell proliferation by gene depletion was examined by MTT assay. In the absence of ionizing radiation, depletion of ELAVL4 alone decreased cell proliferation more than the TMPRSS7 depletion (Fig. 5A). The decrease in cell proliferation was evident after longer incubation time, i.e. more than two days. In the presence of ionizing irradiation, regardless of gene depletion, the cell proliferation decreased as expected from irradiated cells (see the difference in scale of Y-axis of the graph in Fig. 5B). ELAVL4 depletion together with irradiation showed more decrease in cell proliferation when compared to un-irradiated samples. In irradiated samples, proliferation of ELAVL4 depletion decreased more than that of TMPRSS7 depletion. Thus decreased cell proliferation by ELAVL4 depletion might be partly attributable to the enhanced radiation sensitivity as shown in clonogenic assay (Fig. 4C). However, the results of proliferation assay alone do not explain the result of TMPRSS7 clonogenic assay because TMPRSS7 depletion did not affect colony forming ability although the gene depletion slightly decreased cell proliferation. It suggests the presence of additional cellular processes that might affect clonogenic survival. Therefore, we examined cell death, another critical factor for clonogenic survival. Analysis of apoptotic cell death after irradiation revealed that ELAVL4 depletion increased cell death compared to control siRNA-treated samples whereas TMPRSS7 depletion did not (Fig. 5C). Irradiation of TMPRSS7 depleted cells induced comparable cell death to control siRNA-treated samples. Depletion of TMPRSS7 in the absence of irradiation induced less cell death than the control. However, significance of the finding in radiation sensitivity is unclear. Thus taken together, enhanced radiation sensitivity by ELAVL4 depletion seems to be caused by decreased cell proliferation as well as by increased apoptotic cell death. In other words, ELAVL4 is involved in modulation of radiation sensitivity via regulation of cell proliferation and death.

\section{Discussion}

We identified the ELAVL4 gene as a modulator of radiation sensitivity. In the search for the modulator we employed DNA microarray and functional assays including a viability assay. DNA microarray experiment revealed functional categories of genes whose expression is significantly different following ionizing irradiation. More than half of the differentially expressed genes were assigned to the functions such as signal transduction, transport or transcription (Fig. 2). Genes involved in cell cycle, differentiation, proliferation or death belonged to relatively minor categories of the total list. These functional categories of A549 NSCLC cell line are not totally different from those of other cells. For example, $\gamma$-irradiation of Jurkat $\mathrm{T}$ cells revealed 2400 distinct genes that show their involvement in apoptosis, cell proliferation, signal transduction or translation (22). Moreover, genome-wide screening of DNA damage response genes also revealed similar functional categories $(4,7,23)$. These similar functional categories between gene expression profiling and genome-wide screening of modulator of radiation sensitivity may suggest validity of DNA microarray as a possible tool for identification of modulator genes. Our investigation adopted microarray and gene depletion of selected genes to identify modulators of radiation sensitivity. This approach has several advantages compared to large scale screening. First the approach can select genes of specific functional category based on expression levels following irradiation and test their role in radiation sensitivity. Second it can identify modulator genes for radiation sensitivity in a time- and cost-effective way. Third, by combining gene expression profiling and viability assays, the approach may enhance stringency for identification of modulators. As an example, through examination of gene expression alone, this investigation selected several genes for viability assays. The genes were PPP3CA, ELAVL4, TMPRSS7, ZNF239 and CDCA7. Although gene expression levels of these genes were up-regulated, only ELAVL4 depletion exhibited significant radiosensitization (Figs. 3 and 4), only data of ELAVL4 and TMPRSS7 are shown for comparison. Data with other gene depletions did not reveal significant differences in radiation sensitivity. This result suggests that changes of gene expression alone do not guarantee identification of modulators. Rather combination with functional assays enhances the chances of finding cellular radiation modulators. Nonetheless, increased expression following irradiation of genes such as PPP3CA, TMPRSS7, ZNF239 and CDCA7 as well as ELAVL4 suggests possible use of the genes as markers to IR. Since marker genes to IR can be used to assess degree of cellular exposure to IR and even to determine progress of cellular radiation responses by following their expression levels, use of those genes as markers to IR in NSCLC is of importance. Therefore, if not all of the up-regulated genes are found to have activity as modulators of radiation sensitivity, use as potential markers could be expected.

Enhancement of radiation sensitivity by ELAVL4-depletion was attributable to decreased cell proliferation and increased apoptotic cell death (Figs. 4 and 5). ELAVL4 (embryonic lethal, abnormal vision, Drosophila homolog-like 4, alternatively called $\mathrm{HuD}$ ) is an RNA binding protein and associated with neuronal cell maturation, malignant neuroblastoma and Parkinson disease (24-26). In molecular terms, ELAVL4 has been associated with pre-mRNA processing or with enhancement of translation via interaction with eIF4A $(26,27)$. As expected from the critical role of ELAVL4 in those cellular processes, depletion of the gene in A549 cells decreased cell proliferation in the absence of ionizing radiation (Fig. 5A). While the cause of the decreased proliferation is not clear from the result, it is of interest to examine whether effect on pre-mRNA processing or on translation is responsible for the response in A549 NSCLC cells. By comparison, TMPRSS7 depletion resulted in less decrease in cell proliferation (Fig. 5A).

Apoptotic cell death analysis of the depleted cells also revealed difference. Depletion of ELAVL4 induced significantly increased apoptosis following irradiation whereas apoptosis of TMPRSS7-depleted cells was comparable to control which is much less than ELAVL4-depletion (Fig. 5C). Although the exact role of ELAVL4 in DNA damage-induced cell death is not known, the activity of another ELAV family member HuR suggests ELAVL4's possible role in the DNA damage responses (28). HuR protein increases its cytoplasmic abundance after DNA damage and plays roles in stabilization and translation of specific target mRNA (28). This activity may allow transient increase of certain protein products and affect corresponding cellular activities in DNA damage responses. HuR and other 
ELAV family members affect cell growth and proliferation by binding to mRNAs that encode p21, p53, c-myc and c-fos (28). If ELAVL4 protein behaves in such a fashion, increased apoptosis of ELAVL4-depleted cells following DNA damage might have been caused by transient differences in certain proteins that are involved in cell proliferation or death. Although the current data do not provide explanation for possible mechanism of ELAVL4 in DNA damage responses, further understanding of the following may extend our knowledge, and first understanding of the association of DNA damage signaling with expression and activities of ELAVL4 is essential to resolve the issue. DNA damage signaling such as ATM or ATR signaling is known to affect transcription by transducing the signal to many transcription factors such as p53 (29). Thus it is of interest to examine whether promoter of ELAVL4 has cis-regulatory elements for transcription factors that are regulated by DNA damage signaling. In addition, if ELAVL4 is involved in mRNA processing following DNA damage such as ionizing radiation, identification of the specific target mRNAs and their characterization in DNA damage responses should reveal responsible factors for enhanced apoptosis in ELAVL4-depleted cells following irradiation. The role of ELAVL4 in the absence of DNA damage is also a matter of importance. In our data, depletion of ELAVL4 alone decreased cell proliferation (Fig. 5A). Because NSCLC is known for enhanced PI3K/Akt signaling (15), it is of interest to examine whether endogenous function of ELAVL4 is associated with the signaling for cell proliferation. It is also worth examining whether ELAVL4 activity in RNA processing or translation is responsible for the effect on proliferation.

Taken together we identified ELAVL4 as a modulator of radiation sensitivity. Since depletion of ELAVL4 modified radiation sensitivity by affecting cell proliferation and apoptotic cell death, endogenous role of the protein might be associated with regulation of the cellular responses. Induction of ELAVL4 expression following irradiation suggests possible use of the gene as a marker of ionizing irradiation in A549 cells. The capacity for modulation of radiation sensitivity by changing ELAVL4 expression levels could be adopted to enhance radiation cell killing of lung cancer cells. Thus, ELAVL4 is a potential marker and modulator of radiation responses in the A549 lung cancer cell line.

\section{Acknowledgements}

This study was supported by nuclear research and development program of the National Research Foundation of Korea funded by the Korea government (MEST grant code: 20100005406).

\section{References}

1. Sancar A, Lindsey-Boltz LA, Unsal-Kacmaz K and Linn S: Molecular mechanisms of mammalian DNA repair and the DNA damage checkpoints. Annu Rev Biochem 73: 39-85, 2004.

2. Lee JH and Paull TT: Activation and regulation of ATM kinase activity in response to DNA double-strand breaks. Oncogene 26 : 7741-7748, 2007.

3. Zhang D, Zaugg K, Mak TW and Elledge SJ: A role for the deubiquitinating enzyme USP28 in control of the DNA-damage response. Cell 126: 529-542, 2006.
4. Bennett CB, Lewis LK, Karthikeyan G, Lobachev KS, Jin YH, Sterling JF, Snipe JR and Resnick MA: Genes required for ionizing radiation resistance in yeast. Nature Genetics 29: 426-434, 2001.

5. Huang RY, Eddy M, Vujcic M and Kowalski D: Genome-wide screen identifies genes whose inactivation confer resistance to cisplatin in Saccharomyces cerevisiae. Cancer Res 65: 5890$5897,2005$.

6. Westmoreland TJ, Wickramasekara SM, Guo AY, Selim AL, Winsor TS, Greenleaf AL, Blackwell KL, Olson JA Jr, Marks JR and Bennett CB: Comparative genome-wide screening identifies a conserved doxorubicin repair network that is diploid specific in Saccharomyces cerevisiae. PLoS One 4: e5830, 2009.

7. Hurov KE, Cotta-Ramusino C and Elledge SJ: A genetic screen identifies the Triple $\mathrm{T}$ complex required for DNA damage signaling and ATM and ATR stability. Genes Dev 24: 1939-1950, 2010.

8. Chang M, Bellaoui M, Boone C and Brown GW: A genome-wide screen for methyl methanesulfonate-sensitive mutants reveals genes required for $\mathrm{S}$ phase progression in the presence of DNA damage. Proc Natl Acad Sci USA 99: 16934-16939, 2002.

9. Słabicki M, Theis M, Krastev DB, Samsonov S, Mundwiller E, Junqueira M, Paszkowski-Rogacz M, Teyra J, Heninger AK, Poser I, Prieur F, Truchetto J, Confavreux C, Marelli C, Durr A, Camdessanche JP, Brice A, Shevchenko A, Pisabarro MT, Stevanin G and Buchholz F: A genome-scale DNA repair RNAi screen identifies SPG48 as a novel gene associated with hereditary spastic paraplegia. PLoS Biol 8: e1000408, 2010.

10. Jelinsky SA, Estep P, Church GM and Samson LD: Regulatory networks revealed by transcriptional profiling of damaged Saccharomyces cerevisiae cells: Rpn 4 links base excision repair with proteasomes. Mol Cell Biol 20: 8157-8167, 2000.

11. Gasch AP, Huang M, Metzner S, Botstein D, Elledge SJ and Brown PO: Genomic expression responses to DNA-damaging agents and the regulatory role of the yeast ATR homolog Meclp. Mol Biol Cell 12: 2987-3003, 2001.

12. Amundson SA and Fornace AJ Jr: Microarray approaches for analysis of cell cycle regulatory genes. Methods Mol Biol 241: 125-141, 2004.

13. Amundson SA, Bittner M, Chen Y, Trent J, Meltzer P and Fornace AJ Jr: Fluorescent cDNA microarray hybridization reveals complexity and heterogeneity of cellular genotoxic stress responses. Oncogene 18: 3666-3672, 1999.

14. Carmichael J, Degraff WG, Gamson J, Russo D, Gazdar AF, Levitt ML, Minna JD and Mitchell JB: Radiation sensitivity of human lung cancer cell lines. Eur J Cancer Clin Oncol 25: $527-534,1989$.

15. Schuurbiers OC, Kaanders JH, van der Heijden HF, Dekhuijzen RP, Oyen WJ and Bussink J: The PI3-K/AKT-pathway and radiation resistance mechanisms in non-small cell lung cancer. $\mathbf{J}$ Thorac Oncol 4: 761-767, 2009.

16. Brognard J, Clark AS, Ni Y and Dennis PA: Akt/protein kinase $\mathrm{B}$ is constitutively active in non-small cell lung cancer cells and promotes cellular survival and resistance to chemotherapy and radiation. Cancer Res 61: 3986-3997, 2001.

17. Jung IL, Kang HJ, Kim KC and Kim IG: PTEN/pAkt/p53 signaling pathway correlates with the radioresponse of non-small cell lung cancer. Int J Mol Med 25: 517-523, 2010.

18. Kim EJ, Lee SY, Kim TR, Choi SI, Cho EW, Kim KC and Kim IG: TSPYL5 is involved in cell growth and the resistance to radiation in A549 cells via the regulation of p21(WAF1/Cip1) and PTEN/ AKT pathway. Biochem Biophys Res Commun 392: 448-453, 2010.

19. Crnković-Mertens I, Muley T, Meister M, Hartenstein B, Semzow J, Butz K and Hoppe-Seyler F: The anti-apoptotic livin gene is an important determinant for the apoptotic resistance of non-small cell lung cancer cells. Lung Cancer 54: 135-142, 2006.

20. Song L, Coppola D, Livingston S, Cress D and Haura EB: Mcl-1 regulates survival and sensitivity to diverse apoptotic stimuli in human non-small cell lung cancer cells. Cancer Biol Ther 4: 267-276, 2005.

21. Franken NA, Rodermond HM, Stap J, Haveman J and van Bree C: Clonogenic assay of cells in vitro. Nature Protocols 1: 2315-2319, 2006.

22. Park WY, Hwang CI, Im CN, Kang MJ, Woo JH, Kim JH, Kim YS, Kim JH, Kim H, Kim KA, Yu HJ, Lee SJ, Lee YS and Seo JS: Identification of radiation-specific responses from gene expression profile. Oncogene 21: 8521-8528, 2002. 
23. Paulsen RD, Soni DV, Wollman R, Hahn AT, Yee MC, Guan A, Hesley JA, Miller SC, Cromwell EF, Solow-Cordero DE, Meyer T and Cimprich KA: A genome-wide siRNA screen reveals diverse cellular processes and pathways that mediate genome stability. Mol Cell 35: 228-239, 2009.

24. Noureddine MA, Qin XJ, Oliveira SA, Skelly TJ, van der Walt J, Hauser MA, Pericak-Vance MA, Vance JM and Li YJ: Association between the neuron-specific RNA-binding protein ELAVL4 and Parkinson disease. Hum Genet 117: 27-33, 2005.

25. Akamatsu W, Fujihara H, Mitsuhashi T, Yano M, Shibata S, Hayakawa Y, Okano HJ, Sakakibara S, Takano H, Takano T, Takahashi T, Noda T and Okano H: The RNA-binding protein HuD regulates neuronal cell identity and maturation. Proc Natl Acad Sci USA 102: 4625-4630, 2005.
26. Lazarova DL, Spengler BA, Biedler JL and Ross RA: HuD, a neuronal-specific RNA-binding protein, is a putative regulator of $\mathrm{N}$-myc pre-mRNA processing/stability in malignant human neuroblasts. Oncogene 18: 2703-2710, 1999.

27. Fukao A, Sasano Y, Imataka H, Inoue K, Sakamoto H, Sonenberg N, Thoma $\mathrm{C}$ and Fujiwara T: The ELAV protein HuD stimulates capdependent translation in a Poly(A)- and eIF4A-dependent manner. Mol Cell 36: 1007-1017, 2009.

28. Gorospe M: HuR in the mammalian genotoxic response: posttranscriptional multitasking. Cell Cycle 2: 412-414, 2003.

29. Roos WP and Kaina B: DNA damage-induced cell death by apoptosis. Trends Mol Med 12: 440-450, 2006. 